\title{
Ovarian cancer risk assessment in the era of next-generation sequencing
}

\author{
Renata Colombo Bonadio ${ }^{1,2}$, Jéssica Rojas Crespo ${ }^{1}$, Maria Del Pilar Estevez-Diz ${ }^{1,2}$ \\ ${ }^{1}$ Instituto do Cancer do Estado de São Paulo, Faculdade de Medicina do Estado de Sao Paulo, Sao Paulo, Brazil; ${ }^{2}$ Instituto D’Or de Ensino e \\ Pesquisa, Oncologia D’Or, Sao Paulo, Brazil \\ Contributions: (I) Conception and design: All authors; (II) Administrative support: All authors; (III) Provision of study materials or patients: All \\ authors; (IV) Collection and assembly of data: All authors; (V) Data analysis and interpretation: All authors; (VI) Manuscript writing: All authors; (VII) \\ Final approval of manuscript: All authors. \\ Correspondence to: Renata Colombo Bonadio, MD. Instituto do Câncer do Estado de São Paulo, Av. Dr. Arnaldo, 251, São Paulo SP, 01246-000, \\ Brazil. Email: rrccbonadio@gmail.com.
}

\begin{abstract}
Ovarian cancer is one of the cancers most influenced by hereditary factors. Testing for hereditary susceptibility genes is recommended for every woman with epithelial ovarian cancer (EOC). Pathogenic germline variants in $B R C A 1$ and $B R C A 2$ genes are responsible for a substantial fraction of hereditary ovarian cancer. However, alterations in other genes, such as BRIP1, RAD 51C, RAD51D, and mismatch repair genes, also enhance ovarian cancer risk. Other genes may also participate in ovarian carcinogenesis, but their role as ovarian cancer susceptibility genes still needs to be clarified. With several genes involved, the complexity of genetic testing increases. In this context, next-generation sequencing (NGS) allows testing for multiple genes simultaneously, with rapid turn-around time. However, the incorporation of this technology into clinical practice faces some challenges. In this review, we will discuss the ovarian cancer risk assessment in the era of NGS.
\end{abstract}

Keywords: Ovarian cancer; cancer risk; hereditary; next-generation sequencing (NGS)

Submitted Feb 14, 2020. Accepted for publication Jun 20, 2020.

doi: $10.21037 /$ atm-20-1582

View this article at: http://dx.doi.org/10.21037/atm-20-1582

\section{Introduction}

Next-generation sequencing (NGS) is the term used to describe technologies that can sequence multiple genes simultaneously. In the Oncology field, NGS has an enormous contribution to the understanding of tumors biology.

Through tumor samples sequencing, NGS provides somatic mutations profile, allowing a comprehension of their molecular features. Some of these characteristics importantly influence clinical practice nowadays. For instance, patients with ovarian cancer with BRCA mutations or homologous recombination deficiency (HRD) benefit from PARP inhibitors, and immune checkpoint inhibitors have efficacy for tumors with microsatellite instability (1-3). Tumor sequencing may identify germline DNA variants associated with cancer susceptibility. However, some strategies are needed to differentiate between germline and somatic mutations, including analysis with large panels, comparison with normal tissue, and estimations of loss of heterozygosity $(\mathrm{LOH})$ and somatic mosaicism $(4,5)$.

For the assessment of hereditary cancer risk, the sequencing of germline lineage is well-validated. Initially, the traditional Sanger sequencing method usually provided single-genes sequencing. However, hereditary cancer syndromes, such as Hereditary Breast and Ovarian Cancer syndrome, can be explained by germline pathogenic variants

\footnotetext{
^, ORCID: 0000-0001-5818-922X.
} 
in several genes. Consequently, NGS also revolutionized cancer susceptibility genes sequencing, allowing testing of multiple genes simultaneously with fast turn-around time and at lower costs than sequential single-gene testing.

With the rapid integration of these new technologies into clinical practice, health providers should continuously update the knowledge on their applicability and limitations. This review will focus on the genetic risk assessment for ovarian cancer in the era of NGS.

\section{Hereditary ovarian cancer}

A considerable proportion of ovarian cancers are associated with genetic risk. Around 24\% of cases are related to germline mutations in genes known or suspected to be involved in ovarian cancer pathogenesis. Germline BRCA1/2 mutations are the main alterations involved, accounting for $18 \%$ of ovarian cancer cases. Several different genes are responsible for the other $6 \%$ (6).

High-grade serous ovarian cancer (HGSOC) is the most common ovarian cancer histology. A DNA repair mechanism called homologous recombination plays an essential role in this tumor carcinogenesis. Homologous recombination repairs double-strand DNA (dsDNA) breaks efficiently using the sister chromatid as a template. Another mechanism called non-homologous end joining (NHEJ), which also repairs dsDNA break, does not use the sister chromatids as a template and is more error-prone. Thus, when homologous recombination is impaired, it leads to genomic instability, accumulation of DNA errors, and cancer susceptibility. BRCA1 and BRCA2 are involved in the homologous recombination process, as well as other genes implicated in HGSOC, such as RAD S1C, RAD S1D, and BRIP1. Germline mutations, somatic mutations, and methylation of the gene promoter may alter these genes. HRD occurs in approximately half of HGSOC through these different mechanisms (3).

Germline mutations in homologous recombination genes are the main responsible for hereditary HGSOC risk. The impact of mutations on cancer risk is variable according to the gene penetrance. In ovarian cancer, $B R C A 1$ and $B R C A 2$ are high-penetrance genes with carriers of germline mutations presenting an ovarian cancer risk of $39-63 \%$ and $17-27 \%$, respectively (7-11). BRIP1, $R A D 51 C$, and $R A D 51 D$ genes, also involved in homologous recombination, are considered moderate-penetrance genes for ovarian cancer. Lifetime ovarian cancer risk is around $5.2-9 \%$ for $R A D 51 C$ mutations and $10-12 \%$ for $R A D 51 D$ mutations (12-14). Affected women usually develop ovarian cancer at younger ages than those with sporadic cancer. For carriers of BRIP1 mutations, the estimated lifetime risk of ovarian cancer is $5.8 \%(15)$.

HRD has also been reported in ovarian cancer histology other than HGSOC, although in smaller frequencies (3). Moreover, other genes involved in homologous recombination, such as PALB2, ATM, NBN, and CHEK2, may be implied in ovarian carcinogenesis and are frequently included in multigene cancer panels. However, their real effect over ovarian cancer risk is still uncertain.

Finally, low-penetrance genes can also influence ovarian cancer risk. Genome-wide association studies (GWAS) identified single-nucleotide polymorphisms (SNPs) associated with susceptibility for epithelial ovarian cancer (EOC). The 27 loci associated with invasive EOC identified so far accounts for $6.4 \%$ of the polygenic risk for ovarian cancers (16).

Other hereditary cancer syndromes are also associated with ovarian cancer. Lynch syndrome occurs due to germline mutations in genes involved in the mismatch repair system (MLH1, MSH2, MSH6, PMS2). Mismatch repair deficiency or microsatellite instability is identified mainly in endometrioid and clear cell carcinomas, occurring in $10-20 \%$ of these cases (17). However, only a fraction of these cases are due to Lynch syndrome, while others occur due to somatic mutations or epigenetic mechanisms. Women with Lynch syndrome have a lifetime risk of ovarian cancer of $4-12 \%$ (18). In Table 1, we present the frequency of moderate and high-penetrance susceptibility genes for EOC and their associated ovarian cancer risk.

Although less prevalent, some non-EOC $s$ also have their risk enhanced by genetic factors. For instance, germline mutations in DICER1 increase the risk of Sertoli-Leydig cell tumors (19). Germline mutations in STK cause Peutz-Jeghers syndrome and are associated with ovarian sex cord tumors (20).

\section{Criteria for genetic testing}

Current guidelines suggest that all women with EOC should test for ovarian cancer susceptibility genes. This recommendation relies on the observation that many women with pathogenic variants have no other personal or family history that would suggest a hereditary syndrome. In a study with 360 women with ovarian, fallopian tube, or peritoneal carcinoma, among those with germline pathogenic variants, more than $35 \%$ were 65 years or older, and more than $30 \%$ had no family history of ovarian or 
Table 1 Impact of moderate and high-penetrance genes for EOC

\begin{tabular}{|c|c|c|c|c|}
\hline Gene & Main EOC histologic subtype & $\begin{array}{l}\text { Frequency of germline } \\
\text { pathogenic variants (\%) }\end{array}$ & Lifetime risk of EOC (\%) & References \\
\hline$B R C A 1$ & HGSOC & $3-15$ & $39-63$ & $(3,6-10)$ \\
\hline RAD51C & HGSOC & $0-2$ & $5.2-9$ & $(3,6,13,14)$ \\
\hline $\begin{array}{l}\text { MMR genes (MLH1, MSH2, } \\
M S H 6, P M S 2)\end{array}$ & Endometrioid and clear-cell & $0-1$ & $4-12$ & $(3,6,18)$ \\
\hline
\end{tabular}

EOC, epithelial ovarian cancer; HGSOC, high-grade serous ovarian cancer; MMR, mismatch repair.

breast cancer (6).

For EOC patients, guidelines recommend testing for BRCA1, BRCA2, mismatch repair genes (Lynch syndrome), BRIP1, RAD 51C, and RADS1D (21,22). As previously exposed in this review, all these genes are moderate or high-penetrance genes for ovarian cancer risk, and clinical management recommendations are available for them (22). Cascade testing should be offered to relatives of carriers of pathogenic variants.

Nevertheless, no consensus is available on what genetic testing to use, and this decision is based on physicians, genetic counselors, and patients' preferences. Currently, the efficacy and facility of multigene panels make this an attractive choice, and this paper will discuss its advantages and disadvantages.

\section{Gene sequencing interpretation}

Gene sequencing can provide results with different biological meanings. Genetic alteration can be: (I) pathogenic or likely pathogenic; (II) benign or likely benign, or; (III) of uncertain significance. This classification is based on available evidence for each genetic alteration, and the laboratories usually provide these results (23).

A pathogenic variant is known to be associated with cancer risk. A benign variant, otherwise, represents a polymorphism, and the variant has a neutral effect over protein function.

The variant of uncertain significance (VUS) represents the main challenge when interpreting genetic alterations. A VUS result means that, based on current knowledge, it is not possible to say if that variant impacts the protein function or not. Thus, medical management should not change based on this result.
In a large retrospective cohort of individuals who had genetic testing, new evidence reclassified $7.7 \%$ of unique VUS. The reclassification considered the majority (91.2\%) of VUS as benign or likely benign (24). Similarly, in a study of reinterpretation of BRCA1 and BRCA2 VUS, $93.7 \%$ of the reclassified variants were benign or likely benign (25). Despite this, a VUS result can be a great source of distress for patients and their families.

Many tools can help the interpretation of a variant clinical significance $(23,26)$. Variant databases are valuable in providing current evidence. ClinVar (https://www.ncbi.nlm. nih.gov/clinvar/) is an example of a public database where it is possible to share genetic variants found and interpretations of its clinical significance. Another interesting strategy that can aid in the interpretation is the use of computational (in silico) prediction tools. They employ different algorithms to predict missense variants impact on proteins structure and function, and variant effects on splicing. Examples of these tools are PolyPhen2 (http://genetics.bwh.harvard.edu/pph2) and SIFT (http://sift.jcvi.org).

Importantly, the reclassification of variants occurs continuously due to the accumulation of available evidence. Follow-up of VUS carriers is essential as information on their variant may change with time. A global effort to clarify the impact of genetic alterations is ongoing. With this aim, individuals who had a gene panel testing can share their results in registries such as PROMPT (Prospective Registry of MultiPlex Testing) Registry (http://promptstudy.info/).

\section{NGS for cancer risk assessment}

The evolution of NGS turned large genetic testing panels available in clinical practice at affordable prices. Multigene cancer panels test for multiple genes related to hereditary 
Table 2 Gynecologic cancer and cross-cancer panels available from different laboratories (in February 2020)

\begin{tabular}{lll}
\hline Laboratory & Gynecologic panel [number of genes tested] & Cross-cancer panel [number of genes tested] \\
\hline Ambry Genetics & TumorNext-HRD [11] & CancerNext [34] \\
& OvaNext [25] & CustomNext - Cancer [81] \\
GeneDx & Breast/ Gyn Cancer Panel [24] & Comprehensive Common Cancer [47] \\
Invitae & Breast and Gyn Cancers Guidelines-Based [20] & Common Hereditary Cancer [47] \\
& Breast and Gyn Cancers [27] & Multi-Cancer [84] \\
Myriad & - & MyRisk [35] \\
\hline
\end{tabular}

cancer syndrome simultaneously. Thus, as an advantage, they allow for a broad investigation of cancer risk with only one test, at similar prices to that of single-gene testing. Besides, to test multiple genes in a cross-cancer panel costs lower than to test each gene separately. The need for additional tests is not unusual when investigating hereditary cancer after the negative results of single-gene testing. In addition to the higher costs of sequencing isolated genes sequentially, this approach is more time-consuming than a multigene cancer panel (7).

Another positive aspect of multigene panels is that it decreases the chances of missing out a pathogenic mutation. If a limited number of genes are tested based on clinical suspicion and results are negative, mutations in other genes are possible. This concern is especially relevant when family history is limited or in cases of moderate penetrance genes with a less clear clinical phenotype. In a study by Ricker et al., $47.3 \%$ of the mutations identified by multigene panels would have been missed if clinical suspicion guided singlegene tests (27). Similarly, among 708 patients with clinical criteria for hereditary breast and ovarian cancer, Castéra et al. showed that around $40 \%$ of the deleterious mutations detected were in genes other than BRCA1 or BRCA2 (28).

Additionally, a few individuals with hereditary cancer have mosaicism of the pathogenic gene mutation. If the mutation is present at low-levels, Sanger-based sequencing may miss it, while NGS is still efficient in this scenario $(29,30)$.

Nevertheless, several factors should be considered when using panels. Patients should be aware of the range of information that multigene panels can provide. One disadvantage of the broader panels is that, for some test results, no guidelines and little literature are available on how to manage the patient. Notably, management for some low- and moderate-penetrance genes is unclear. Moreover, for some genes included in multigene panels, a lack of enough information on specific cancer risks may occur.
Patients should be aware of this possibility during pre-test counseling. On the other hand, a better comprehension of gene variants' clinical significance may soon be available as we test more patients and collect more information.

Another disadvantage of NGS cancer panels is the higher rates of VUS detection. The VUS rates of multigene panels range from $19.7 \%$ to as high as $88 \%$ (31-33). In comparison, VUS rates in a BRCA1/2 alone testing and a breast-cancer panel were $<1 \%$ and $14 \%$, respectively, in a recent report (34). Once again, patients should be prepared for the possibility of this result when performing gene sequencing.

\section{Gynecological and cross-cancer panels}

Many gynecological and cross-cancer panels are currently available at clinical practice. Gynecological panels may focus on susceptibility genes for ovarian cancer alone, ovarian, and endometrial cancer, or ovarian and breast cancer. Cross-cancer panels are broader and include most or all of the known susceptibility genes for hereditary cancer. Some of the cross-cancer panels, such as CustomNextCancer from Ambry Genetics, allow flexibility to choose which genes the test will include.

The choice between each test will depend on patients and health providers' preferences, considering the advantages and disadvantages discussed previously. In Table 2, we list some of the gynecologic cancer panels and the cross-cancer panels available from different laboratories.

Despite numerous panels options, access to these tests varies hugely depending on its availability in public and private settings in different countries (35).

\section{Upfront tumor sequencing}

Another important topic to consider is how the 
investigation of germline and somatic mutations should start. Tumor sample testing is important for ovarian cancer treatment nowadays since it can provide information on the existence of somatic BRCA1/2 mutations and HRD, which are predictors of response to platinum agents and $P A R P$ inhibitors. Of note, phase III studies evaluating the efficacy of $P A R P$ inhibitors for ovarian cancer explored these biomarkers differently (36-41).

Tumor sequencing can identify mutations (germline or somatic) in homologous recombination genes (3). Additionally, HRD leads to the occurrence of genomic scars, represented by the $\mathrm{LOH}$, large-scale transitions, and telomeric allele imbalance. A test that evaluates these three types of alterations and a test of $\mathrm{LOH}$ alone have been validated as predictors of $\operatorname{HRD}(42,43)$.

Tumor sample testing can provide all this information: HRD status and gene mutations (germline or somatic). If a mutation is identified, the sequencing of the normal cells is required to determine if the mutation is germline or somatic. However, if mutations in susceptibility genes are ruled out by tumor sequencing, additional testing could potentially be avoided. Otherwise, when initial healthy cell sequencing discards germline pathogenic variants, tumor testing is still required since somatic mutations (or HRD status) can influence treatment decisions (44).

Considering all this, in situations where information on germline and somatic mutations are necessary for patient management, starting with tumor sequencing may decrease the need for double testing (tumor testing and germline testing). For BRCA1 and BRCA2 genes, for instance, tumor sequencing would identify mutations in around $20-30 \%$ of HGSOC cases, and these individuals (20-30\%) would need additional germline testing. Otherwise, germline sequencing would identify $B R C A 1$ and $B R C A 2$ pathogenic variants in $18-20 \%$ of the cases; around $80 \%$ would need additional tumor testing to evaluate BRCA1 and BRCA2 somatic mutations (or HRD status). Thus, upfront tumor sequencing could be an attractive alternative in these situations in which knowing the tumor mutational profile is relevant for clinical practice.

A multinational guideline suggests the use of tumor sequencing to evaluate both germline and somatic BRCA1/2 mutations (44). However, guidelines from Oncology Societies, including the American Society of Clinical Oncology (ASCO) and the European Society of Medical Oncology (ESMO), provides a different recommendation $(45,46)$. ASCO and ESMO guidelines suggest germline testing first, followed by somatic tumor testing for patients who do not carry a germline pathogenic variant. ASCO guideline also highlights that although trials of ovarian cancer treatment stratified patients using HRD assays, they currently make no recommendations to support its routine use (46).

One reason to recommend upfront germline testing is the variable accuracy of tumor sequencing. DNA obtained from the blood has high quality, and germline sequencing methods are well-validated and accurate. Tumor sequencing accuracy, otherwise, is influenced by several technical factors, especially for large genes such as $B R C A$. For tumor sequencing, formalin-fixed, paraffin-embedded (FFPE) or fresh frozen specimens are preferred if available (44). Fresh frozen specimens provide DNA with high quality but are less available than FFPE specimens. The percentage of tumor cells in the sample should preferably be high (at least three times the limit of detection), and NGS is the recommended tumor sequencing method (44). Capoluongo et al. provide a series of recommendations to improve tumor sequencing accuracy (44).

Fortunately, when proper technical standards are applied, high success rates have been reported with tumor sequencing. In a study with 114 patients with HGSOC and a $B R C A 1 / 2$ pathogenic/likely pathogenic variant, tumor BRCA1/2 NGS testing had an accuracy of $97 \%$ compared to Sanger sequencing for germline mutations (47). Similarly, in a study by Fumagalli et al., with 23 EOC patients with $B R C A 1 / 2$ pathogenic/ likely pathogenic variants, tumor NGS testing was able to identify all cases of germline $B R C A 1 / 2$ pathogenic/ likely pathogenic variants (48).

Despite its high accuracy, germline sequencing also has limitations. A pitfall of germline sequencing recently described in another hereditary syndrome, the Li-Fraumeni syndrome (LFS), is the aberrant clonal expansion (ACE) phenomenon. LFS occurs due to germline TP53 pathogenic variants. In the ACE phenomenon, clonal populations with somatic TP53 mutations may be detected in the blood or saliva, confounding the germline testing results. With its high efficacy, NGS may detect even a small fraction of mutant alleles, resulting in a wrong conclusion of LFS (49). The impact of this phenomenon in other hereditary cancer syndromes is less known.

\section{Pre- and post-test counseling}

Previously, pre-test counseling addressed a discussion of the impact and management of the specific syndrome investigated. The complexity of genetic counseling 
increased considerably with multigene panels. During pretest counseling in the NGS era, individuals should receive information about all the range of results that can be provided by the panel.

A valuable strategy for genetic counseling is to group the genes into categories to help patients understand the extent of possible results. One classification proposed is the following: high penetrance genes with management guidelines available; moderate penetrance genes with little consensus on appropriate medical management and; genes for which the degree of cancer risk is still not well understood (50).

Genetic counseling should address the possibility of identifying cancer risk for different primary sites and the management strategies available or not for each of them. A discussion of the meaning of VUS and the high VUS rates expected is helpful to diminish the anxiety caused by these results. With proper pre-test counseling and information on the advantages and disadvantages of different genetic sequencing tests, patients can participate actively in the test choice, with an informed decision.

Post-test counseling will be guided by the results found. Recommendations for cascade relatives testing should be included in this stage, when appropriate. Since information on variants changes with the accumulation of new evidence, patients should be followed and notified about updates, such as VUS reclassifications and new clinical management guidelines (50).

\section{Conclusions}

Genetic risk has a crucial impact on ovarian cancer and is associated with at least one-fourth of ovarian cancer cases. The evolution of NGS allows a rapid evaluation of multiple cancer susceptibility genes at similar costs to single-gene sequencing. However, these broader panels are associated with some challenges. For some genes included, the ovarian cancer risk is not clear, and no medical management guidelines are available. Additionally, VUS rates increase with more genes tested, and information on variants pathogenicity is continuously generated. Thus, patients should be aware of all these aspects before the ordering of hereditary cancer panels. Besides, the complexity of multigene panels requires health providers' proper training and updating.

\section{Acknowledgments}

Funding: None.
Provenance and Peer Review: This article was commissioned by the Guest Editors (Stergios Boussios and Nicholas Pavlidis) for the series "Ovarian Cancer: State of the Art and Perspectives of Clinical Research" published in Annals of Translational Medicine. The article was sent for external peer review organized by the Guest Editors and the editorial office.

Conflicts of Interest: All authors have completed the ICMJE uniform disclosure form (available at http://dx.doi. org/10.21037/atm-20-1582). The series "Ovarian Cancer: State of the Art and Perspectives of Clinical Research" was commissioned by the editorial office without any funding or sponsorship. RCB has received financial support for educational programs from AstraZeneca and financial support for attending symposia from Roche, outside the submitted work. The other authors have no other conflicts of interest to declare.

Ethical Statement: The authors are accountable for all aspects of the work in ensuring that questions related to the accuracy or integrity of any part of the work are appropriately investigated and resolved.

Open Access Statement: This is an Open Access article distributed in accordance with the Creative Commons Attribution-NonCommercial-NoDerivs 4.0 International License (CC BY-NC-ND 4.0), which permits the noncommercial replication and distribution of the article with the strict proviso that no changes or edits are made and the original work is properly cited (including links to both the formal publication through the relevant DOI and the license). See: https://creativecommons.org/licenses/by-nc-nd/4.0/.

\section{References}

1. Le DT, Uram JN, Wang H, et al. PD-1 Blockade in Tumors with Mismatch-Repair Deficiency. N Engl J Med 2015;372:2509-20.

2. Diaz LA, Le D, Maio M, et al. Pembrolizumab in microsatellite instability high cancers: Updated analysis of the phase II KEYNOTE-164 and KEYNOTE-158 studies. Ann Oncol 2019;30:v475-32.

3. da Cunha Colombo Bonadio RR, Fogace RN, Miranda $\mathrm{VC}$, et al. Homologous recombination deficiency in ovarian cancer: a review of its epidemiology and management. Clinics 2018;73:e450s.

4. Schrader KA, Cheng DT, Joseph V, et al. Germline 
Variants in Targeted Tumor Sequencing Using Matched Normal DNA. JAMA Oncol 2016;2:104-11.

5. Parsons DW, Roy A, Plon SE, et al. Clinical tumor sequencing: an incidental casualty of the American College of Medical Genetics and Genomics recommendations for reporting of incidental findings. J Clin Oncol 2014;32:2203-5.

6. Walsh T, Casadei S, Lee MK, et al. Mutations in 12 genes for inherited ovarian, fallopian tube, and peritoneal carcinoma identified by massively parallel sequencing. Proc Natl Acad Sci U S A 2011;108:18032-7.

7. Price KS, Svenson A, King E, et al. Inherited Cancer in the Age of Next-Generation Sequencing. Biol Res Nurs 2018;20:192-204.

8. Easton DF, Ford D, Bishop DT. Breast and ovarian cancer incidence in BRCA1-mutation carriers. Breast Cancer Linkage Consortium. Am J Hum Genet 1995;56:265-71.

9. Ford D, Easton DF, Stratton M, et al. Genetic heterogeneity and penetrance analysis of the BRCA1 and BRCA2 genes in breast cancer families. The Breast Cancer Linkage Consortium. Am J Hum Genet 1998;62:676-89.

10. Mavaddat N, Peock S, Frost D, et al. Cancer risks for BRCA1 and BRCA2 mutation carriers: results from prospective analysis of EMBRACE. J Natl Cancer Inst 2013;105:812-22.

11. van Asperen CJ, Brohet RM, Meijers-Heijboer EJ, et al. Cancer risks in BRCA2 families: estimates for sites other than breast and ovary. J Med Genet 2005;42:711-9.

12. Loveday C, Turnbull C, Ramsay E, et al. Germline mutations in RAD51D confer susceptibility to ovarian cancer. Nat Genet 2011;43:879-82.

13. Loveday C, Turnbull C, Ruark E, et al. Germline RAD51C mutations confer susceptibility to ovarian cancer. Nat Genet 2012;44:475-6; author reply 6.

14. Song H, Dicks E, Ramus SJ, et al. Contribution of Germline Mutations in the RAD51B, RAD51C, and RAD51D Genes to Ovarian Cancer in the Population. J Clin Oncol 2015;33:2901-7.

15. Ramus SJ, Song H, Dicks E, et al. Germline Mutations in the BRIP1, BARD1, PALB2, and NBN Genes in Women with Ovarian Cancer. J Natl Cancer Inst 2015;107:djv214.

16. Phelan CM, Kuchenbaecker KB, Tyrer JP, et al. Identification of 12 new susceptibility loci for different histotypes of epithelial ovarian cancer. Nat Genet 2017;49:680-91.

17. Lynch HT, Casey MJ, Snyder CL, et al. Hereditary ovarian carcinoma: heterogeneity, molecular genetics, pathology, and management. Mol Oncol 2009;3:97-137.
18. Pal T, Permuth-Wey J, Sellers TA. A review of the clinical relevance of mismatch-repair deficiency in ovarian cancer. Cancer 2008;113:733-42.

19. Schultz KAP, Williams GM, Kamihara J, et al. DICER1 and Associated Conditions: Identification of At-risk Individuals and Recommended Surveillance Strategies. Clin Cancer Res 2018;24:2251-61.

20. Young RH, Dickersin GR, Scully RE. A distinctive ovarian sex cord-stromal tumor causing sexual precocity in the Peutz-Jeghers syndrome. Am J Surg Pathol 1983;7:233-43.

21. Randall LM, Pothuri B, Swisher EM, et al. Multidisciplinary summit on genetics services for women with gynecologic cancers: A Society of Gynecologic Oncology White Paper. Gynecol Oncol 2017;146:217-24.

22. NCCN guidelines. Genetic/familial high-risk assessment: Breast and ovarian. Version 1.2020. Accessed 11 Jun 2020. Available online: http://www.nccn.org/professionals/ physician_gls/pdf/genetics_screening.pdf

23. Richards S, Aziz N, Bale S, et al. Standards and guidelines for the interpretation of sequence variants: $a$ joint consensus recommendation of the American College of Medical Genetics and Genomics and the Association for Molecular Pathology. Genet Med 2015;17:405-24.

24. Mersch J, Brown N, Pirzadeh-Miller S, et al. Prevalence of Variant Reclassification Following Hereditary Cancer Genetic Testing. JAMA 2018;320:1266-74.

25. So MK, Jeong TD, Lim W, et al. Reinterpretation of BRCA1 and BRCA2 variants of uncertain significance in patients with hereditary breast/ovarian cancer using the ACMG/AMP 2015 guidelines. Breast Cancer 2019;26:510-9.

26. Wallis Y, Payne S, McAnulty C, et al. Practice guidelines for the evaluation of pathogenicity and the reporting of sequence variants in clinical molecular genetics. Association for Clinical Genetic Science and the Dutch Society of Clinical Genetic Laboratory Specialists; 2013. Available online: https://www.acgs.uk.com/media/10791/ evaluation_and_reporting_of_sequence_variants_bpgs_ june_2013_-_finalpdf.pdf

27. Ricker C, Culver JO, Lowstuter K, et al. Increased yield of actionable mutations using multi-gene panels to assess hereditary cancer susceptibility in an ethnically diverse clinical cohort. Cancer Genet 2016;209:130-7.

28. Castéra L, Krieger S, Rousselin A, et al. Next-generation sequencing for the diagnosis of hereditary breast and ovarian cancer using genomic capture targeting multiple candidate genes. Eur J Hum Genet 2014;22:1305-13.

29. Friedman E, Efrat N, Soussan-Gutman L, et al. Low- 
level constitutional mosaicism of a de novoBRCA1 gene mutation. Br J Cancer 2015;112:765-8.

30. Rohlin A, Wernersson J, Engwall Y, et al. Parallel sequencing used in detection of mosaic mutations: comparison with four diagnostic DNA screening techniques. Hum Mutat 2009;30:1012-20.

31. Selkirk CG, Vogel KJ, Newlin AC, et al. Cancer genetic testing panels for inherited cancer susceptibility: the clinical experience of a large adult genetics practice. Fam Cancer 2014;13:527-36.

32. Kurian AW, Hare EE, Mills MA, et al. Clinical evaluation of a multiple-gene sequencing panel for hereditary cancer risk assessment. J Clin Oncol 2014;32:2001-9.

33. Slavin TP, Niell-Swiller M, Solomon I, et al. Clinical Application of Multigene Panels: Challenges of NextGeneration Counseling and Cancer Risk Management. Front Oncol 2015;5:208.

34. Wishart G, Payne S, Allen Z, et al. Is the variant of uncertain significance (VUS) rate important in genetic testing for breast cancer? Eur J Surg Oncol 2018;44:874-5.

35. Prapa M, Solomons J, Tischkowitz M. The use of panel testing in familial breast and ovarian cancer. Clin Med (Lond) 2017;17:568-72.

36. Mirza MR, Monk BJ, Herrstedt J, et al. Niraparib Maintenance Therapy in Platinum-Sensitive, Recurrent Ovarian Cancer. N Engl J Med 2016;375:2154-64.

37. González-Martín A, Pothuri B, Vergote I, et al. Niraparib in Patients with Newly Diagnosed Advanced Ovarian Cancer. N Engl J Med 2019;381:2391-402.

38. Pujade-Lauraine E, Ledermann JA, Selle F, et al. Olaparib tablets as maintenance therapy in patients with platinumsensitive, relapsed ovarian cancer and a BRCA1/2 mutation (SOLO2/ENGOT-Ov21): a double-blind, randomised, placebo-controlled, phase 3 trial. Lancet Oncol 2017;18:1274-84.

39. Moore K, Colombo N, Scambia G, et al. Maintenance Olaparib in Patients with Newly Diagnosed Advanced Ovarian Cancer. N Engl J Med 2018;379:2495-505.

40. Coleman RL, Oza AM, Lorusso D, et al. Rucaparib maintenance treatment for recurrent ovarian carcinoma after response to platinum therapy (ARIEL3): a

Cite this article as: Bonadio RC, Crespo JR, Estevez-Diz MDP. Ovarian cancer risk assessment in the era of next-generation sequencing. Ann Transl Med 2020;8(24):1704. doi: 10.21037/atm20-1582 randomised, double-blind, placebo-controlled, phase 3 trial. Lancet 2017;390:1949-61.

41. Coleman RL, Fleming GF, Brady MF, et al. Veliparib with First-Line Chemotherapy and as Maintenance Therapy in Ovarian Cancer. N Engl J Med 2019;381:2403-15.

42. Abkevich V, Timms KM, Hennessy BT, et al. Patterns of genomic loss of heterozygosity predict homologous recombination repair defects in epithelial ovarian cancer. Br J Cancer 2012;107:1776-82.

43. Telli ML, Timms KM, Reid J, et al. Homologous Recombination Deficiency (HRD) Score Predicts Response to Platinum-Containing Neoadjuvant Chemotherapy in Patients with Triple-Negative Breast Cancer. Clin Cancer Res 2016;22:3764-73.

44. Capoluongo E, Ellison G, López-Guerrero JA, et al. Guidance Statement On BRCA1/2 Tumor Testing in Ovarian Cancer Patients. Semin Oncol 2017;44:187-97.

45. Paluch-Shimon S, Cardoso F, Sessa C, et al. Prevention and screening in BRCA mutation carriers and other breast/ ovarian hereditary cancer syndromes: ESMO Clinical Practice Guidelines for cancer prevention and screening. Ann Oncol 2016;27:v103-10.

46. Konstantinopoulos PA, Norquist B, Lacchetti C, et al. Germline and somatic tumor testing in epithelial ovarian cancer: ASCO guideline. J Clin Oncol 2020;38:1222-45.

47. Dougherty BA, Lai Z, Hodgson DR, et al. Biological and clinical evidence for somatic mutations in BRCA1 and BRCA2 as predictive markers for olaparib response in high-grade serous ovarian cancers in the maintenance setting. Oncotarget 2017;8:43653.

48. Fumagalli C, Tomao F, Betella I, et al. Tumor BRCA Test for Patients with Epithelial Ovarian Cancer: The Role of Molecular Pathology in the Era of PARP Inhibitor Therapy. Cancers 2019;11:1641.

49. Weitzel JN, Chao EC, Nehoray B, et al. Somatic TP53 variants frequently confound germ-line testing results. Genet Med 2018;20:809-16.

50. Fecteau H, Vogel KJ, Hanson K, et al. The evolution of cancer risk assessment in the era of next generation sequencing. J Genet Couns 2014;23:633-9. 